\title{
Visual angle as a determinant of perceived interobject distance
}

\author{
CHARLES A. LEVIN \\ Baldwin-Wallace College, Berea, Ohio \\ and \\ RALPH NORMAN HABER \\ University of Illinois, Chicago, Illinois
}

\begin{abstract}
Two new experiments and a reanalysis of Toye's (1986) data are used to examine the relationship between true distance and perceived distance in natural scenes. In the first experiment, 8 subjects estimated 78 interobject distances, formed by all pairs of 13 objects, while viewing the objects from a fixed position. The results showed that estimated distance is a linear function of the visual angle between objects as well as of the true distance. This relationship results in distances perpendicular to the line of sight being overestimated in relation to true distances and to distances parallel to the line of sight. These findings were confirmed by reanalysis of a comparable data set from Toye. Since changes in the visual angle can come about through changes in alignment with the line of sight, viewing distance, or interobject distance, Experiment 2 was designed to determine whether the visual angle effect was due to one of these, or whether it was an independent effect. In Experiment 2, 8 subjects estimated six interobject distances from 12 viewing positions. The results showed that visual angle predicted estimated distance independently of how the change in visual angle came about, suggesting that the greater the visual angle between objects, the more their separation is overestimated.
\end{abstract}

Most research on the perception of natural scenes has been concerned with describing how accurately we perceive the distance from objects to ourselves (e.g., Gilinsky, 1951; Gogel, 1969, 1972, 1974). This has led to theories of space perception based on egocentric distances and directions, and to a nearly exclusive focus on research concerning the variables that affect egocentric distances (see Gogel, 1977).

What has been lacking is work on the variables that affect how we perceive and report the distance between objects (exocentric distance) located in a scene, and the corresponding development of a theory of the perceived layout of space (see Haber, 1986). Until recently, no one had measured the perceived shape or layout of space. In two recent studies, however, researchers have used natural scenes to investigate the perceived layout of space.

Toye (1986) used a scene that consisted of 13 randomly placed objects in an open field. He required his subjects to estimate all interobject distances with the use of three different methods: absolute estimation, map drawing, and triadic comparisons. Toye's subjects made each estimate twice, with half of them making the second judgment from a new viewing position. Toye's work resulted in three

\footnotetext{
Parts of this research were reported in a master's thesis by the first author. We would like to thank Rebecca Burzette for her assistance in running the second experiment. Partial support for this research was provided by Grant NEI-07801 from the National Eye Institute to the second author. Correspondence should be addressed to R. N. Haber, 3268 Summit Avenue, Highland Park, IL 60035.
}

major findings: (1) observers had a very accurate representation of a visual scene, regardless of the method of estimation; that is, the scaled judgments showed a strong visual and statistical similarity to the real scene; (2) the shape of the perceived scene changed as the viewing position changed; and (3) this distortion in the shape of the perceived scene occurred because the observers systematically estimated the interobject distances that were closely aligned with the line of sight (egocentric) differently from those that were oriented more perpendicularly to the line of sight. Toye suggested that the difference was due to an underestimation of the interobject distances aligned with the line of sight as compared with those perpendicular to the line of sight, although he did not speculate whether the former were underestimated absolutely, or the latter were overestimated absolutely.

Wagner (1985), who used a scene similar to Toye's (1986), required his subjects to estimate interobject distances with the use of three methods: magnitude estimation, category estimation, and mapping. He also asked his subjects to estimate the size of the angle, as well as the area formed by triads of stakes, by using the methods of magnitude estimation and perceptual matching. Using an affine compression model, he concluded that there is a distortion in the perception of space. Wagner, like Toye, claimed that this distortion was due to a relative compression of interobject distances aligned with the light of sight.

Although Toye (1986) and Wagner (1985) each found a distortion in the perception of the shape of a scene, neither was able to explore the causes of such differences, 
primarily because they both placed the objects in the scenes randomly. Such placement prevented direct tests of equal distances at different orientations to the line of sight, since Wagner and Toye did not have a sufficient number of such contrasts in their data.

In the present study, we report two new experiments, along with a reanalysis of Toye (1986), in an attempt to investigate three issues in the perception of the distances between objects in a scene.

First, is there a difference in the perception of interobject distances that differ in their alignment with the observer's line of sight?

Second, what variables might account for the differences produced by amount of alignment with the line of sight? We manipulated two. The first concerned the visual angle between a pair of objects being judged from a particular viewpoint. If the interobject distance is held constant, as alignment with the line of sight decreases from $0^{\circ}$ to $90^{\circ}$, the visual angle between the two objects with respect to the viewer increases as well. Is visual angle a predictor of error in estimating distance? The second variable concerned the difference between two kinds of interobject distances, both of which are aligned with the line of sight. Egocentric aligned distances arise when the observer judges the distance between himself/herself and an object. Exocentric aligned distances arise when the observer judges the distance between two objects that are both located along the line of sight.

Third, given answers to the preceding questions, what is the quantitative relationship between true and perceived distance, and what is the weighting of predictor variables?

In addition to addressing these quantitative questions, the new experiments afforded the opportunity to replicate the results of Toye with respect to global accuracy and the effect of viewing position.

\section{EXPERIMENT 1}

\section{Methods}

Subjects. Eight male graduate students with no previous experience of psychophysical experiments volunteered as observers. The observers were screened for normal binocular vision and acuity, both by self-report. Each observer was tested twice. They were randomly assigned to one of two groups. In the no-position-change group, Time 1 and Time 2 testings occurred at the same viewing position. In the position-change group, Time 1 and Time 2 testings occurred at different viewing positions.

Stimuli. The visual scene in this experiment was related to the one used by Toye (1986). It contained 13 stakes, each extending straight up $1.5 \mathrm{~m}$ out of the ground. Each stake was topped with a rectangular sign bearing a printed capital letter from " $A$ " through "M." The stakes were planted in a flat grassy area approximately $20 \mathrm{~m}$ in diameter. The field surrounding the stimulus area was approximately four times larger and was bounded by tall buildings and trees.

The stimulus positions were chosen according to specific criteria, to provide control over the visual angle and alignment with the line of sight of the interobject distance separating each pair of objects; both the visual angle and the alignment were defined with respect to the two viewing positions of the subjects. The visual angle required for viewing any pair of objects is the angle, taken at the position of the observer, between the two lines of sight when the observer looks first at one of the objects and then at the other. To produce a range of visual angles, we selected stake placements that varied in alignment with the line of sight of the viewer. The degree of alignment with respect to the line of sight of an interobject distance is defined by the angle formed between the line of sight of the subject and a line segment connecting the two objects. A $0^{\circ}$ alignment defines a pair of objects whose interobject distance is parallel to the line of sight. A $90^{\circ}$ alignment defines a pair of objects whose interobject distance is perpendicular to the line of sight.

Two stakes served as viewing positions (labeled " $A$ " and " $B$ "'; see Figure 1). These two positions were chosen so that all stimuli were located in front of the observers; the two positions were $90^{\circ}$ rotations of each other.

The remaining stakes were located so that each stake when viewed from one position had a corresponding stake when viewed from the other viewing position. Correspondence was satisfied by two criteria: the distance from one of the viewing positions to the stake equaled the distance from the other viewing position to its corresponding stake; and a stake and its corresponding stake were at the same distance from the axes that went through their respective viewing positions. For example, Stake $L$ was $38.5 \mathrm{ft}(11.73 \mathrm{~m})$ from Viewing Position $A$ and $23.4 \mathrm{ft}(7.13 \mathrm{~m})$ from the $x$-axis; its corresponding stake, $\mathrm{G}$, was $38.5 \mathrm{ft}$ from Viewing Position $\mathrm{B}$ and $23.4 \mathrm{ft}$ from the $y$-axis.

An infinite number of points could fulfill these two criteria for stake position selection. To select 11 from among these, the stimulus area was divided into a Cartesian coordinate grid with the origin at the center of the stimulus area. Pairs of stake positions were then selected which met the criteria as corresponding positions and maximized the number of interobject distances that could be included in the following three groups: (1) egocentric aligned, in which one stake position was the viewing position and another was chosen so that the interobject distance was close to parallel with the line of sight considered from one viewing point and close to perpendicular to the line of sight considered from the other viewing position and its corresponding distance was the reverse (e.g., Distances AI and BK, respectively); (2) exocentric aligned, in which neither stake position was a viewing position, and the interobject distance was

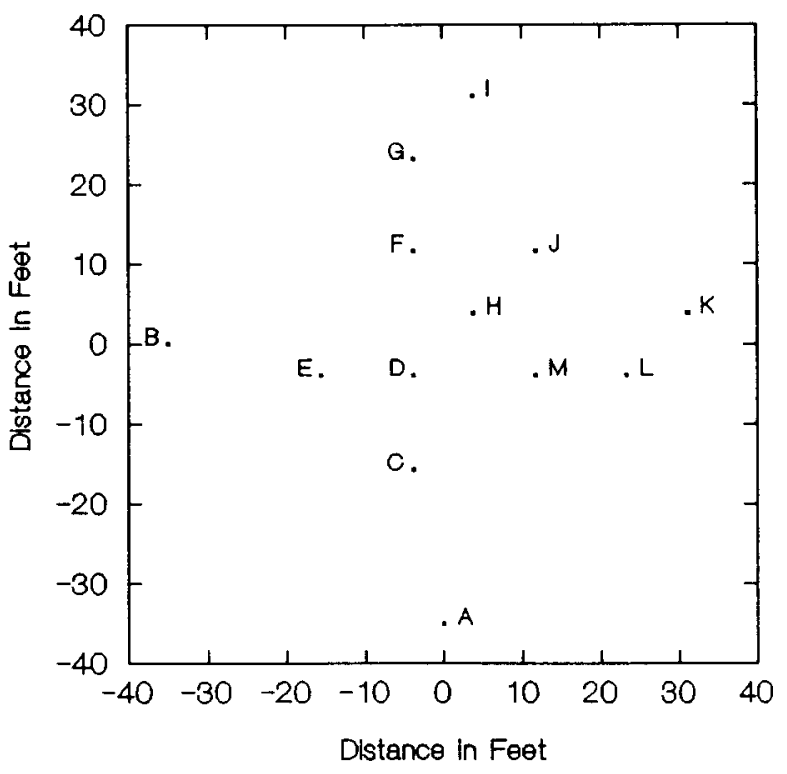

Figure 1. Map of the layout of the 13 stakes in Experiment 1. 
close to parallel with the line of sight when considered from one viewing position and close to perpendicular to the line of sight when considered from the other viewing position (e.g., Distances GC and LE, respectively); and (3) horizontal, in which neither stake was a viewing position, and the interobject distance was close to perpendicular to the line of sight with respect to one viewing position and parallel to the line of sight with respect to the corresponding viewing position (e.g., Distances FK and IL, respectively). Stake placements were made to maximize the number of the parallel and perpendicular interobject distances according to these criteria.

Procedure. Four observers were tested at the same time: 2 seated next to the A stake and 2 seated next to the B stake. All observations were conducted on sunny days when illumination was well within the photopic range. The observers were given a clipboard as a writing surface, a pen, and a list of the 78 pairs of the 13 stakes in a random order with a line next to each letter pair (e.g., "MI _."). The instructions to the observers were the following: "For each pair of objects, I want you to estimate the distance between them in feet, and write that estimate on the appropriate line on the answer sheet. You should remain seated, though you can otherwise move your head and body freely."

Each observer made all 78 judgments twice, with a rest period of 15-30 min between judging periods. The observers in the noposition-change group made both sets of judgments from the same viewing position, 2 observers from Viewing Position $A$ and 2 from Viewing Position $\mathbf{B}$. The observers in the position-change group made one set of judgments from each viewing position: 2 made their first set of judgments from Position $\mathrm{A}$, and 2 made their first set of judgments from Position B.

\section{Results}

The relation of true to estimated distance judgments. To provide a picture of the overall results, the mean estimated distance (based on the average of two estimates from each of 8 subjects) is plotted in Figure 2 as a function of the true distance for each of the 78 distances. As can be seen, the relationship is linear, nearly perfect, with a slight tendency for overestimation. The values on which

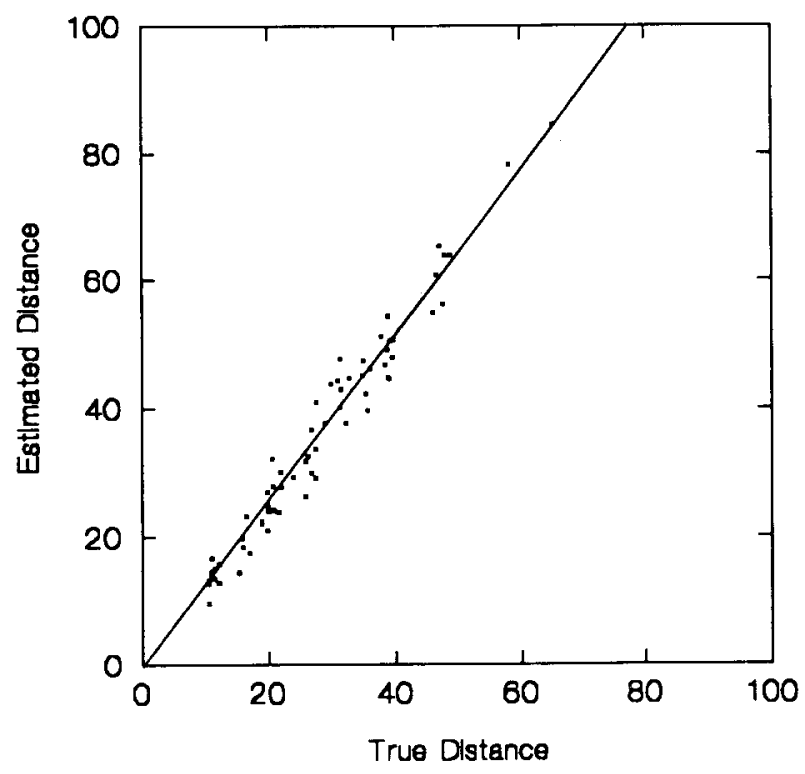

Figure 2. Mean estimated distance (based on averaging two estimations made by 8 subjects) as a function of the true distance, for each of 78 interobject distances. this plot is based, along with the standard deviations about each of the 78 means, are given in the Appendix.

To examine the metric underlying each observer's distance estimates, 16 nonlinear regression analyses relating the 78 estimated distances to true distances were computed, 1 from each of the two testing times for each of the 8 subjects. The regression equation used was

$$
\mathrm{ED}=I+B(\mathrm{TD})^{e},
$$

where ED is the estimated distance in feet, $I$ is a constant describing the intercept of the function with the true distance axis, $B$ is a regression coefficient, TD is the true distance in feet, and $e$ is an exponent.

Each of the 16 equations was examined for the contribution of the predictor terms. None of the 16 intercept constants was different from zero $(p>.05)$, and none of the 16 exponents was different from unity $(p>.05)$.

These terms were therefore deleted from the model, and linear regression equations were recomputed. The mean $R^{2}=0.933[S E=0.093 ; F(1,77)=503.12, p<.001]$, and the mean regression coefficient $B=1.11$ ( $S E=$ 0.031 ). The regression coefficient was significantly above unity for every subject $(p<.05)$.

The results suggest that the observers, as a group, cannot be distinguished from an ideal observer using a ratio scale of distance: the relation of estimated to true distance is a linear function that passes though the origin. Furthermore, virtually all of the variance in the estimates is accounted for by the true distances being judged. The regression coefficient shows that, on the average, the observers overestimated interobject distances by about $10 \%$.

Effect of visual angle. The regression equations were recomputed with the use of a nonlinear model, adding the visual angle variable. Each interobject distance was specified in terms of the visual angle, which ranged from $0^{\circ}$ (for the egocentric perfectly aligned interobject distances) to $82^{\circ}$. Because the intercept did not differ from zero in any of the 16 regression equations, and because none of the exponents differed from unity, the equations were again recomputed by using the following linear model:

$$
\mathrm{ED}=B(\mathrm{TD})+G(\mathrm{VA}),
$$

where $G$ is the regression coefficient on visual angle (VA), and $B$ is the regression coefficient on true distance (TD). Averaged over the 16 regression equations, the mean $R^{2}=0.972[F(2,76)=14.60, p<.001]$; the mean $B=$ $1.108(S E=0.031)$, which was significantly greater than unity $(t=3.48, p<.001)$; and the mean $G=0.164$ $(S E=0.045)$, which was significantly greater than zero $(t=3.64, p<.001)$. The two regression coefficients were significantly different from unity and zero, respectively, for each subject at each testing time.

Figure 3 is a graph of the effects of visual angle for the 78 estimated distances, averaged across the two trials for the 8 subjects. The abscissa plots the visual angles of the interobject distance, which ranged from $0^{\circ}$ to $82^{\circ}$. The ordinate plots the mean ratio of estimated divided by true distance, in which unity indicates perfect estimation, values above unity indicate overestimation (e.g., a ratio 


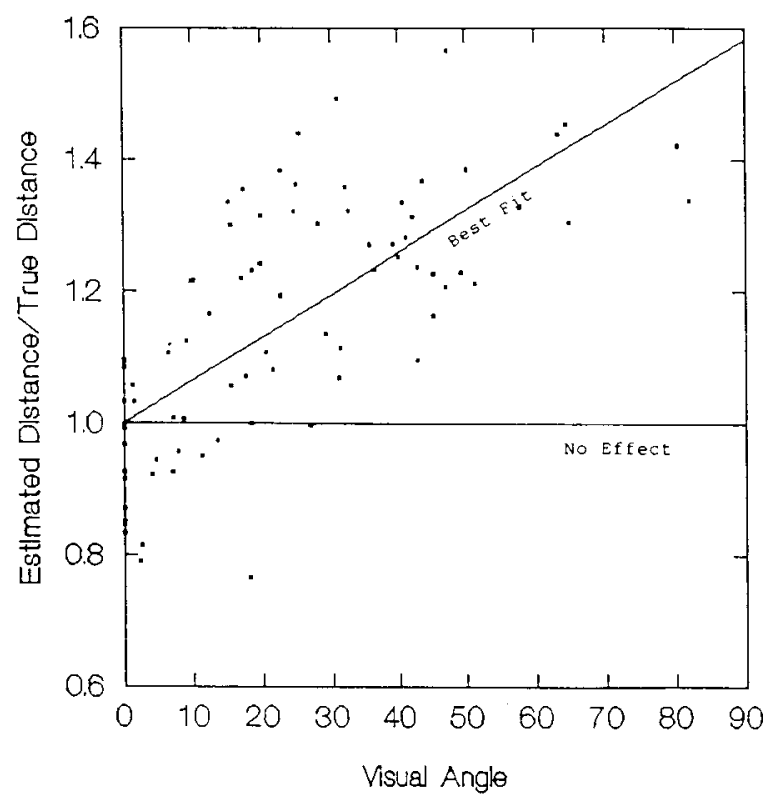

Figure 3. Change in the amount of overestimation (ratio of estimated to true distance) as a function of the visual angle of the interobject distance being estimated. Each of the 78 data points is the mean of the estimates summed over 8 observers and two trials.

of 1.2 indicates a $20 \%$ overestimation of distance), and values below unity indicate underestimation. A best-fit linear function is shown through the 78 points; there was a steady increase in the influence of visual angle over the range investigated.

These results show that visual angle is a significant predictor of estimated distance: with true distance held constant, the larger the visual angle between two objects, the larger the estimated distance to be between them. For example, consider interobject distance $\mathrm{AI}$, which has a true distance of $66 \mathrm{ft}(20.12 \mathrm{~m})$. When $\mathrm{AI}$ is viewed from Position $A$, its visual angle is $0^{\circ}$, and it is overestimated by an average of $11 \%-74.6 \mathrm{ft}(22.74 \mathrm{~m})$. Its corresponding distance BK (also $66 \mathrm{ft}$ ), when viewed from Position $\mathrm{A}$, has a visual angle of $82^{\circ}$, and is overestimated on the average by nearly $30 \%-85.4 \mathrm{ft}(26.03 \mathrm{~m})$. Considered another way, the regression equation states that, with true distance held constant, as the visual angle of a fixed interobject distance is increased by each $10^{\circ}$, its estimated distance is increased by $1.6 \mathrm{ft}(0.48 \mathrm{~m})$.

Egocentricity versus exocentricity. To determine whether there was a difference in the accuracy of estimating aligned egocentric in comparison with aligned exocentric distances (e.g., AD vs. CF; BD vs. EM; etc.), a $t$ test was performed on the amount of error for the two kinds of aligned distances. Each distance estimate was subtracted from its corresponding true distance and divided by true distance to yield an error proportionate to the true distance being judged. There was no difference in proportion of error for these two distance types $\left(t_{24}=.14, p=\right.$ .886). There was also no difference in the best-fit function for these two types of distances. For this scene, the perception of interobject distance aligned with the line of sight is not affected by egocentricity.

Reanalysis of the data of Toye. Toye (1986) reported his data only in terms of the alignment of each interobject distance vis-à-vis the line of sight of the observer. We obtained the stake placements used by Toye and computed the visual angles for each interobject distance. We also obtained the 16 distance estimation matrices ( 2 from each of Toye's 8 observers) and performed a regression analysis employing true distance, adding visual angle as a new predictor variable.

As in the present experiment, all 16 nonlinear equations produced intercepts that were insignificantly different from zero, and exponents that were insignificantly different from unity. Therefore, we computed the linear regression equation:

$$
\mathrm{ED}=B(\mathrm{TD})+G(\mathrm{VA}) .
$$

The mean $R^{2}=0.930$; mean $B=0.860(S E=0.074)$, which was not significantly different from unity $(t=1.88$, $p>.10)$; and mean $G=0.175(S E=0.041)$, which was significantly greater than zero $(t=4.27, p<.001)$. None of these three terms was significantly different from those obtained in the present experiment $(p>.05)$. Considering each subject separately, visual angle was a significant predictor $(p<.05)$ for 13 of the 16 matrices.

Hence, even though Toye (1986) did not examine it, his data reveal a significant effect of visual angle, of the same magnitude as that found in the present experiment.

Replication of Toye's scaling of space. Toye (1986) used multidimensional scaling (MDS) to demonstrate that his observers used their distance estimates consistently, and that when scaled in two dimensions, those estimates located each of the stakes in virtually the same configuation as was present in the actual scene being perceived (see Kosslyn, Pick, \& Fariello, 1974, for a comparable example). To determine whether the present results replicated Toye's findings, comparable MDS analyses were carried out on the present data. Toye used only an ordinal level of specification; we used both an ordinal and a ratio level. In no case did the two levels differ statistically. We report the ordinal level only.

Analysis of the 16 distance dissimilarity matrices (2 from each observer) yielded scaled scenes locating the objects that were adequately described by two dimensions. Examination of Shepard diagrams (see Kruskal \& Wish, 1978) revealed no nonlinearities imposed on the data by the scaling.

Like Toye (1986), we examined two measures from the MDS scaling: the consistency of the observers' estimations, and the accuracy with which the locations of the objects in the scaled scene matched those in the real scene.

The consistency analysis uses iterated stress, a measure of how well an observer's judgments can be scaled to fit a two-dimensional space. The MDS-scaled scenes accounted for the subjects' estimates with a good degree of accuracy - a mean final iteration stress of only 0.046 . Kruskal and Wish's (1978) criterion suggests that mean 
final iteration stress this low represents a very good fit. Furthermore, there was no difference in final iteration stress between the two conditions $[t(30)=1.981, p=$ $.068]$ or between the two testing periods $[t(30)=1.219$, $p=.243$ ]. Analysis of variance revealed no interaction between time and condition $[F(1,12)=.046, p=.832]$.

The accuracy analysis uses mean uniterated stress as a measure of goodness of fit between the location of objects in the scaled scene and those in the true scene. Zero iteration stress is determined by using the actual locations of the stakes and calculating the initial stress in the MDS solutions before any iterations are made. This amount of stress is indicative of how well the scaled locations approximate the true locations. The mean zero iteration stress was 0.127 , a good fit by the Kruskal and Wish criterion. As with final iteration stress, there was no difference in goodness of fit between the two conditions [ $t(30)$ $=.049, p=.962]$, or between the two times of testing $[t(30)=.847, p=.411]$. Analysis of variance revealed no interaction between condition and time $[F(1,12)=$ $.081, p=.781]$.

The effect of position change was assessed by an MDS analysis of the similarity in the zero iteration stress of the scaling of the two sets of estimates, contrasting the subjects who changed positions between the two sets of estimates with those who did not change position (Time 2 zero iteration stress when the initial configuration consists of Time 1 scaled coordinates). The subjects in the no-position-change condition had a mean reliability goodness of fit of 0.106 , whereas the subjects in the positionchange condition had a significantly poorer mean reliability goodness of fit of $0.215[t(14)=2.64, p=.038]$.

Each of these findings replicated results in Toye (1986).

\section{Discussion}

The two most important findings in this experiment concern the quantitative nature of the distortion of the shape of a perceived scene, and the role of visual angle in accounting for this distortion.

Quantitative relationship between real and perceived distance. This experiment replicated the work of Toye (1986) and Wagner (1985), in that the relationship between real distance and estimated distance was found to be linear. This is consistent with other research conducted under similar conditions. In a review of 70 studies of Stevens's exponents for the estimation of distances between objects in a rich and full scene, Wiest and Bell (1985) reported that for direct viewing the exponent is not significantly different from one. (They also reported that when distances are estimated from memory or are estimated by inference-e.g., distance between continents-the exponents are significantly lower than one. They showed too that when the scenes being judged were impoverished of good information about depth, exponents were likely to be less than unity as well.)

This experiment also showed that the perception of egocentric aligned distances is comparable to the perception of exocentric aligned distances. In a review essay, Gogel
(1977) reported a compression of egocentric distances in relation to exocentric distances. However, in the studies that he considered, researchers failed to control for the degree of visual angle and included exocentric distances where the near object occluded the far object, which would cause the observer to move his/her head in order to view both objects. In the present experiment, in which we controlled for visual angle and prevented occlusion, no difference between these two types of distances was found.

The role of visual angle in accounting for the distortion of perceived space. We examined the hypothesis that the errors in the estimation of interobject distances perpendicular to the line of sight could be attributed to the visual angle between the two objects being judged. The results show that as the visual angle increases, the magnitude of error in estimation increases linearly. This error will not have any impact on judgments of distances among objects that are arranged in parallel with the observer's line of sight: there is little visual angle between such pairs of objects. However, the error introduced by the visual angle effect can be quite large for the distance between two objects that are equidistant from the ob-
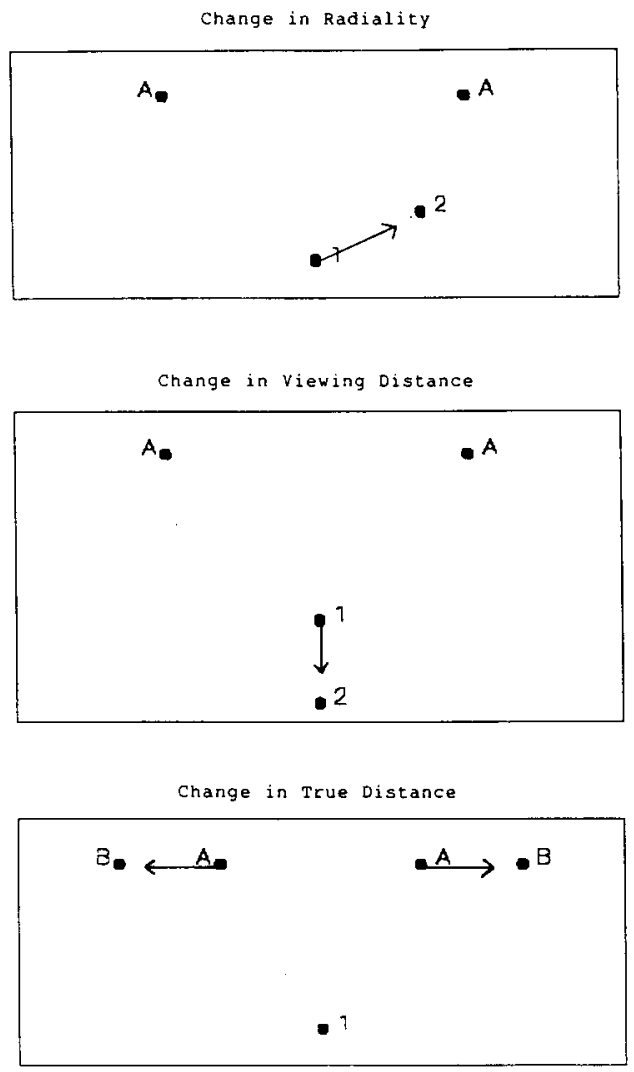

Figure 4. Three illustrations of how visual angle can change: due to a change in radiality as the observer shifts to the side (top); due to a change in viewing distance, as the observer shifts closer (middle); due to a change in true distance between the objects, without any shift in position of the observer (bottom). In the drawings, $A$ and $B$ represent two objects; and 1 and 2, two positions of an observer. 
server, an arrangement that can produce large visual angles. The net result of this asymmetry is that visual extents along the horizontal dimension of space will be perceived to be larger than they actually are. (See the General Discussion for further justification of this conclusion.)

If we consider the geometry of the present experimental scene, we find that variation in visual angle can arise from three different sources (see Figure 4): (1) a change in alignment with respect to the line of sight-a given interobject distance of fixed mean viewing distance can be viewed from a position with a different degree of alignment; (2) a change in viewing distance - a given interobject distance of fixed alignment with respect to the line of sight can be observed from different viewing distances; and (3) a change in true distance - the distance between two objects can change, with alignment to the line of sight and viewing distance held constant.

In the present data, we have found evidence that visual angle predicts estimations of distance when true distance is held constant. However, since visual angle is obviously correlated by principles of geometry with alignment to the line of sight, with true distance, and with viewing distance, as shown in Figure 4, a somewhat different set of constraints governing the placement of objects is necessary for one to differentiate among these variables.

\section{EXPERIMENT 2}

Experiment 2 was designed with new stake positions and a new procedure, so that each interobject distance could be estimated from many different viewing positions, each of which would produce different combinations of alignment, viewing distance, and visual angle.

The viewing positions were selected so that comparisons across positions would yield a set of distances to be judged in which alignment with the line of sight was a constant and viewing distance changed, as well as a set in which viewing distance was constant and the alignment varied. This allowed a test of visual angle as a predictor of estimated distance when true distance was held constant, alignment was held constant, or viewing distance was held constant. If visual angle is a significant predictor of estimated distance when true distance is a constant, there is de facto evidence that true distance alone is not the determiner of the visual angle effect. Furthermore, if visual angle predicts estimated distance when true distance and alignment or true distance and viewing distance are held constant, the variables of alignment and viewing distance can also be eliminated as independent determiners of the visual angle effect.

\section{Method}

Subjects. Eight male undergraduates served as observers. They participated as partial fulfillment of the requirements of an introductory psychology course, and they had not served in Experiment 1. All observers had normal binocular vision and visual acuity.

Stimuli. The distance estimations were made in the field used in Experiment 1. The observers estimated the distance between two vertical 5 -ft stakes held by two experimenters. At the beginning of each estimation trial, the two experimenters stood together; then they both backed up until they were at a predetermined distance apart that produced the distance to be estimated. Six different distances were used: $10,20,35,45,60$, and $75 \mathrm{ft}(3.05,6.10,10.67$, $13.72,18.92$, and $22.86 \mathrm{~m}$ ). Each observer made estimates of these six distances while standing at each of 12 different viewing positions, for a total of 72 distance estimations.

The 12 viewing positions are illustrated in Figure 5, spread throughout an area roughly $12 \times 25 \mathrm{~m}$. Each viewing position differed in its alignment and viewing distance (which was specified as the distance from the observer to the midpoint of the interobject distance between the two objects). From a single viewing position, all six interobject distances to be estimated had the same alignment and the same viewing distance. The viewing positions were arranged so that no stake was occluded by any other viewing position. The subjects made their judgments while standing on a marker, but they could otherwise move their heads and bodies.

The 12 viewing positions to be occupied by the observers were each identified by $0.23-\mathrm{m}$ squares of cardboard on the ground (labeled 1-12, respectively). Smaller markers embedded below the level of the grass were invisible to the subjects; these identified the locations where the two experimenters placed the stakes for each sequence.

Procedure. All 8 observers were tested in the same session, which lasted $1 \mathrm{~h}$. The observers were given a clipboard and a pen. Each clipboard held 12 sheets of paper, 1 for each of the 12 viewing positions, denoted at the top with a letter (1-12), and followed by six blank lines, one for each of the six estimates to be made from each position. The order of the sheets was randomized for each subject, with the constraint that only 1 subject was allowed to make observations from any given viewing position on any given trial.

The subjects were told that they were to estimate 72 distances, 6 different ones from each of 12 different viewing positions. The

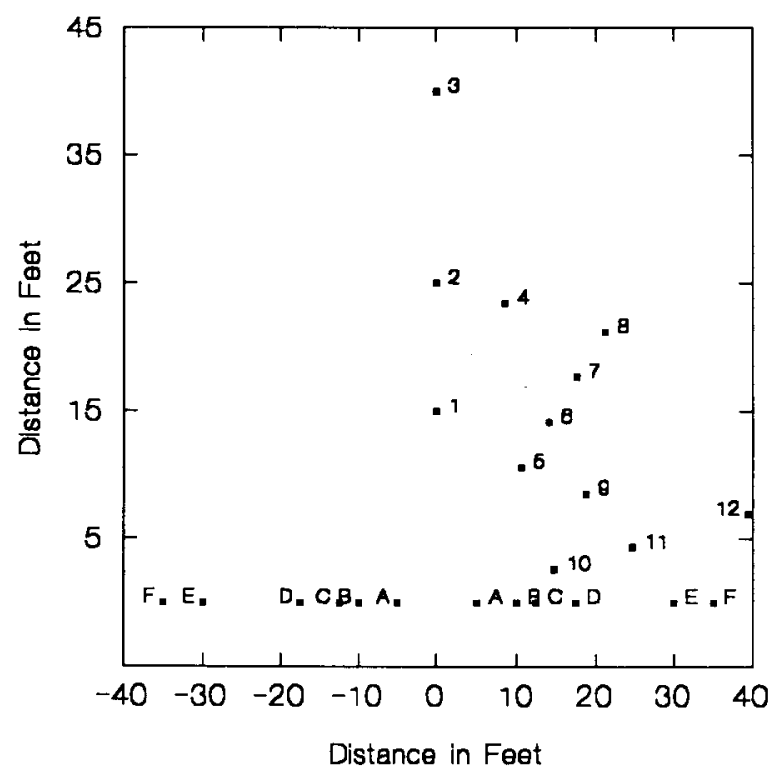

Figure 5. Map of the layout of the six distances and 12 viewing positions for Experiment 2. The alignment with the line of sight (in degrees), and viewing distance (in feet and meters) for all of the six distances estimated from each of the 12 positions are as follows: (1) $90^{\circ}, 15 \mathrm{ft}(4.57 \mathrm{~m})$; (2) $90^{\circ}, 25 \mathrm{ft}(7.62 \mathrm{~m}) ;(3) 90^{\circ}, 40 \mathrm{ft}$ (12.19 m); (4) $70^{\circ}, 25 \mathrm{ft}(7.62 \mathrm{~m})$; (5) $45^{\circ}, 5 \mathrm{ft}$ (1.52 m); (6) $45^{\circ}$, $20 \mathrm{ft}(6.10 \mathrm{~m}) ;(7) 45^{\circ}, 25 \mathrm{ft}(7.62 \mathrm{~m}) ;(8) 45^{\circ}, 30 \mathrm{ft}(9.14 \mathrm{~m})$; (9) $20^{\circ}, 25 \mathrm{ft}(7.62 \mathrm{~m}) ;(10) 10^{\circ}, 15 \mathrm{ft}(4.57 \mathrm{~m})$; (11) $10^{\circ}, 25 \mathrm{ft}$ (7.62 $\mathrm{m})$; and (12) $10^{\circ}, 40 \mathrm{ft}(12.19 \mathrm{~m})$. 
instructions for making the estimations were the same as in Experiment 1 . In addition, the subjects were told that this was an experiment on perception and not on memory and that they were to write down their distance estimates as accurately as possible each time, without regard to what they had written down for previous distances.

Each of the 12 sets of trials began with the 8 observers standing at their respective viewing positions, given by the letter printed on the top sheet. The two experimenters stood at the center of the field. When the observers indicated they were ready, the two experimenters walked away from each other until they reached the required locations for them to place their respective stakes for that trial. They stood to the outside, with each stake placed on the proper buried marker. When each observer had indicated a response on the sheet, the experimenters walked back to the center, turned, and then walked off the next distance. The random order of the distance presentation was varied for each of the 12 trials.

When all six distances from the first viewing position had been estimated, the subjects put that scoring sheet on the bottom of the stack and moved to the viewing position written on the top of the next sheet, and the next set of six estimations then began. This procedure continued until all subjects had estimated 72 distances ( 6 distances at each of 12 viewing positions).

\section{Results}

The data from this experiment were analyzed with the use of linear regression models in order to determine whether visual angle was a predictor of estimated distance with three combinations of trials: when true distance was constant, when true distance and alignment were constant, and when true distance and viewing distance were constant. In determining the effect of alignment and viewing distance, true distance is also held constant because true distance is a prima facie determiner of estimated distance. Because only six different true distances were employed, we did not attempt to examine complete best-fit regression models. In all cases, the regression model tested was

$$
\mathrm{ED}=I+G(\mathrm{VA}) \text {. }
$$

1. To determine whether visual angle predicts estimated distance only when the magnitude of the true distance varies, best-fit models were estimated by subject, by true distance. In these data, visual angle varied with changes in alignment, changes in viewing distance, or both, but not with changes in true distance, since distance was held constant.

It was found that visual angle significantly predicted estimated distance for all subjects, for each of the six distances. The mean $R^{2}=0.887[F(1,5)=106.05, p<$ $.001]$, mean $I=0.03(S E=0.02)$, which was not significantly different from zero $(t=1.55, p>.20)$; and $G=0.480(S E=0.025)$, which was significantly greater than zero $(t=19.2, p<.001)$.

This indicates that even when true distance is a constant, if visual angle is allowed to vary by changing alignment, viewing distance, or both, there is a systematic variation in estimated distance. Hence, true distance is not a geometrically confounded determinant of visual angle.

2 . To determine whether visual angle predicts estimated distance only when the alignment of the interobject distance varies with respect to the line of sight, best-fit models were estimated by subject, by true distance, for constant alignment. In these data, visual angle varied with changes in viewing distance, but not with changes alignment or true distance.

It was found that visual angle predicted estimated distance for all subjects for all distances and all alignments. The mean $R^{2}=0.937[F(1,4)=134.00, p<.001]$; mean $I=0.02(S E=0.015)$, which was not significantly different from zero $(t=1.33, p>.20)$; and mean $G=$ $0.478(S E=0.026)$, which was significantly greater than zero $(t=18.38, p<.001)$.

This indicates that when true distance and alignment are constant and visual angle is allowed to vary with changes in viewing distance, there is still a systematic prediction of estimated distance by visual angle. Hence, alignment is not a geometrically confounded determinant of visual angle.

3. To determine whether visual angle predicts estimated distance only when the viewing distance varies, best-fit models were estimated by subject, by true distance, for constant viewing distance. In these data, visual angle varied with changes in alignment, but not with changes in viewing distance or true distance.

It was found that visual angle predicted estimated distance for all subjects for all true distances and all viewing distances. The mean $R^{2}=0.968[F(1,3)=372.0$, $p<.001]$; mean $I=0.001(S E=0.0008)$, which was not significantly different from zero $(t=1.25, p>.20)$; and mean $G=0.455$ ( $S E=0.020$ ), which was significantly greater than zero $(t=22.75, p<.001)$.

This indicates that when true distance and viewing distance are constant and visual angle is allowed to vary with changes in alignment, there is still a systematic change in estimated distance. Hence, viewing distance is not a geometrially confounded determinant of visual angle.

The coefficients $G$ for visual angle were not different from each other among the three equations above $(p>.05)$.

\section{Discussion}

The results of the three regression equations, taken together, show that variations in visual angle predict variations in estimated distance, independently of variation in true distance, alignment, or viewing distance. Although the latter three variables are confounded in natural scenes, when their combinations are separated and controlled experimentally, the effects of visual angle persist.

Thus, the visual angle effect from Experiment 1 is not the result of a correlated change in true distance, alignment, or viewing distance. Rather, a change in visual angle resulting from a change in any of these variables produces a change in estimated distance.

There is a methodological concern. The procedure of asking subjects to estimate the same six interobject distances from each of the 12 viewing positions raises the possibility that the subjects' responses were more consistent than would have been expected if different distances had been used for each viewing position. Several aspects of the design and results mitigate against this concern. First, subjects would have had to become aware that there 
were repetitions among the distances. This would have been difficult, since the markers for the experimenters telling them where to place the stakes were hidden from the subjects' view, the grassy field was evenly textured, the median distance from a subject to a stake was $6 \mathrm{~m}$, with the largest distance over $18 \mathrm{~m}$, and the subjects could not see the estimates they had made from previously occupied viewing positions. Second, no subject commented on the repetition during the postexperimental debriefing and questioning. Third, if the repetitious distance estimates had been constrained by subjects' remembering their earlier estimates, the effect of visual angle should have been smaller in the second experiment than in the first. In both experiments, visual angle varied from trial to trial, but in the second experiment, that variation was possibly constrained by memory for the repetition of the same distances. However, the regression coefficient $G$, while significant in both experiments, was smaller in Experiment 1 (not larger as demanded by this methodological concern). For all these reasons, the repetition of distances does not seem to have been a source of confounding.

\section{GENERAL DISCUSSION}

Six new or extended findings of these studies follow:

1. The perception of the distance between objects is slightly distorted as a function of the visual angle between the objects.

2. This distortion produces an overestimation of interobject distances perpendicular to the line of sight, but it has little effect on those parallel to the line of sight, making a scene appear to be more elliptical along its horizontal axis (the one perpendicular to the line of sight) than it actually is.

3. Because this distortion occurs along only one axis of a scene, it accounts for why there is a change in the perceived relationship among the objects in a scene when there is a change in viewing position.

4. There is no difference in the perception of egocentric distances and exocentric radial distances.

5 . The relationship between estimated and true distance is linear (over the range and conditions tested here).

6. Observers' estimates of the distances among objects in a scene can be used to construct a multidimensionally scaled map of the scene, a map that is highly consistent with the distance estimates, and that closely resembles the actual scene, although it is one that displays a systematic distortion. Hence, this procedure provides a measure of an observer's perceived layout of space, one that detects accuracies as well as inaccuracies.

Although these experiments show that any of the variables that affect the visual angle between the objects being viewed will affect estimated distance, they do not indicate why increasing the visual angle produces more overestimation of distance. The geometric analysis implicit in Figure 4 suggests three potential causes of the visual angle effect.

First, change in the degree of alignment of a pair of objects with the line of sight (holding interobject distance and viewing distance constant) alters the amount of distance information available to the observer. For example, an interobject distance perfectly perpendicular to the line of sight has no gradient in texture across the ground between the two objects: the density of the texture is the same around both objects, so texture variation is not correlated with interobject distance. Therefore, texture gradient information becomes progressively unavailable when interobject distances approach perpendicularity with the line of sight. Similar logic shows that several other important sources of information about distances between objects are reduced in quality when the objects are equidistant from the observer (see Künnapas, 1968). If texture and other sources of information are reduced or unavailable, perhaps viewers are forced to rely on other less veridical information, information that is correlated with the magnitude of the visual angle.

Second, change in the viewing distance from the objects (holding alignment and true distance constant) alters the degree of visual angle in a quite different way. For example, if an observer standing $15 \mathrm{~m}$ from the midpoint of an interobject distance moves closer to be only $7.5 \mathrm{~m}$ from that midpoint, the visual angle between the two objects increases by one third. However, under the assumption that the distance information about near objects is better than that about more distant ones, it is counterintuitive that moving the viewing position closer to the objects should increase the inaccuracy that underlies the visual angle effect. If anything, moving closer to the objects should improve the accuracy of estimation of interobject distance. Consequently, it seems unlikely that changes in viewing distance itself can account for the visual angle effect.

And finally, to change the real interobject distance (with alignment and viewing distance held constant) changes visual angle. But the observers in these experiments, as well as those of Toye (1986) and Wagner (1985), were quite accurate in estimating interobject distances closely aligned with the line of sight over a range extending from near reach to over $25 \mathrm{~m}$, so it seems unlikely that observers would experience difficulty in estimating interobject distances perpendicular to the line of sight over the same range unless another variable was introduced. Furthermore, no nonlinearity in the function relating real to estimated interobject distance was detected for any kind of interobject distance in this (or Toye's) study. Hence, it seems unlikely that the magnitude of the interobject distance accounts for the visual angle effect.

Of the three explanations considered, the visual angle effect seems most likely to be related to a degradation in spatial information specifying the separation of objects that are equidistant from the observer. But this explanation also implies that observers replace the missing visual spatial information with nonvisual information that is correlated with visual angle. It can be speculated that this might be some extraretinal signal specifying the amount of head and/or eye turning needed to look from one object to another when one is judging their separation (see Haber \& Hershenson, 1980 , for a discussion). Extraretinal signals have been included by many theorists to help explain the perception 
of the third and/or fourth (movement) dimensions of space, but they have received less attention in accounting for the first two dimensions, those being examined here. The present results indicate that the role of extraretinal signals in the perception of horizontal distance should be examined further (e.g., Cutting, 1986).

Another aspect of visual angle concerns the amount of scene that can be picked up in a single glance. When the angle is small, as with pairs of objects closely aligned with the line of sight, no eye movement would be needed to see both objects in a single glance. With a greater visual angle, an eye movement might be needed. Haber and Hershenson (1980), considering Sander's (1963) work, suggested that for a task such as this one, a separation of $20^{\circ}$ would be likely to trigger an eye movement. Such logic would imply a discontinuous function of visual angle and error in estimating distance. However, the present data strongly suggest that visual angle is linearly related to error in distance estimation, so that it is the magnitude of an eye movement, not the probability of its occurrence, that is relevant.

It is ironic that distances perpendicular to the line of sight are the ones most erroneously estimated. One of the oldest assumptions in space perception theories, dating to those of Helmholtz (see Haber, 1986), is that the horizontal dimensions of space are accurately perceived because they are specified in retinal coordinates. The radial depth dimension, on the other hand, because there is no retinal "signal" for depth, has to be inferred or extracted, and therefore is less accurate. Although these views are still commonly held, the present results cast substantial doubt on their reasonableness.

All the manipulations in these experiments, as well as their results, have been interpreted as aspects of distance and space perception, not in terms of size perception. Although it is true that the interobject "distance" between two separated objects in space is logically equivalent to the "size" of the empty space between them, it seems unreasonable and unlikely that the two are perceptually equivalent. It is especially unlikely, considering that the 5 - $\mathrm{ft}$ tall objects were only an inch in cross-section, but the empty spaces between them were as long as $25 \mathrm{~m}$. Principles of perceptual grouping and figure/ground apply strongly here: the stakes were separate skinny objects, with empty spaces between them that varied in distance. The objects were not the boundary edges of long objects in which the spaces between the edges define the objects' size.

Size information is sometimes invoked with respect to distance by treating object height as a dimension of size. The relation of the tops of the objects to their visual intersection with the horizon can provide information about their distance from the viewer (see Sedgwick, 1986), especially if the viewer can assume that all the objects have the same height. However, this is not likely to be a useful cue in the present experiment: the 1.5-m-high stakes were viewed from about a $1.5-\mathrm{m}$ eye height, so there was little useful correlation between the distance of an object from a viewer and the height of the intersection of the top of an object with the horizon. Furthermore, the field in which the stakes were viewed was bordered by mature trees between 10 and $20 \mathrm{~m}$ in height, about $60 \mathrm{~m}$ away. Thus, there was no horizon "line" against which to line up the heights of the objects.

Given these assumptions, we have ignored issues of size perception in this context.

Finally, in the discussion of Experiment 1, it was concluded that visual extents along the horizontal dimension of space (those perpendicular to the line of sight) are perceived to be longer than they actually are. Furthermore, by implication, the radial dimension of space aligned with the line of sight are perceived accurately. These are much stronger claims than merely concluding that horizontal dimensions are overestimated in relation to radial dimensions. These claims imply a one-to-one relationship between verbal estimations in feet and physical distances in feet: the observer's internalized metric concept of the unit foot is equal to that of the physical foot.

Evidence of a ratio scale underlying the observers' estimates is not sufficient by itself to support the assumption of the equality of the internalized and physical metric. For example, if all subjects had an internalized metric that was on the average $10 \%$ smaller than the physical unit foot, then, on the basis of the present data, horizontal dimensions of space would be accurately estimated and radial dimensions would be underestimated.

Even if this possibility is accepted, the within-subject analyses presented in these experiments (as well as those of Toye, 1986) make a more compelling case for the absolute magnitude conclusions offered here. Each subject showed the same underlying ratio scale of distance, the same ratio of estimations of the horizontal to the radial dimensions of space, and the same quantitative distortion in the horizontal dimension as a function of visual angle. These commonalities would be unlikely if each subject had a different relationship between an internalized and a physical metric. Hence, although not definitive, the present results support the claim of an absolute overestimation of the horizontal dimension of space and an accurate estimation of the radial dimension.

\section{REFERENCES}

Cutting, J. E. (1986). Perception with an eye for motion. Cambridge, MA: MIT Press.

GiLinsky, A. S. (1951). Perceived size and distance in visual space. Psychological Review, 58, 460-487.

GOGEL, W. C. (1969). The absolute and relative size cues to distance. American Journal of Psychology, 82, 228-234.

Goofe., W. C. (1972). Scalar perceptions with binocular cues to distance. American Journal of Psychology, 85, 477-497

Gocel, W. C. (1974). Cognitive factors in spatial responses. Psychologica, 17, 213-225.

Gocie L, W. C. (1977). A metric of visual space. In W. Epstein (Ed.), Stability and constancy in visual perception (pp. 129-182). New York: Wiley.

HABER, R. N. (1986). Toward a theory of the perceived layout of scenes In A. Rosenfeld (Ed.), Human and machine vision I/ (pp. 109-148) New York: Academic Press.

HABER, R. N., \& Hershenson, M. (1980). The psychology of visual perception (2nd ed.). New York: Holt, Rinehart \& Winston.

Kosslyn, S. M., Pick, H. L.. \& FArIello, G. R. (1974). Cognitive maps in children and men. Child Development, 45, 707-716 
KruSKal, J. B., \& Wish, M. (1978). Multidimensional scaling. London: Sage University Papers.

KüNNAPAS, T. (1968). Distance perception as a function of available visual cues. Journal of Experimental Psychology, 77, 523-529.

SANDERS, A. (1963). The selective process in the functional visual field. Soesterberg, The Netherlands: Institute for Perception RVO-TNO.

SEDGWICK, H. A. (1986). Space perception. In R. Boff, L. Kaufman, \& J. P. Thomas (Eds.), Handbook of perception and human performance (Vol. 1, pp. 21-1 to 21-57). New York: Wiley.

TOYE, R. C. (1986). The effect of viewing position on the perceived layout of space. Perception \& Psychophysics, 40, 85-92.

Wagner, M. (1985). The metric of visual space. Perception \& Psychophysics, 38, 483-495.

WIEST, W. M., \& BELl, B. (1985). Stevens's exponent for psychophysical scaling of perceived, remembered, and inferred distance. Psychological Bulletin, 98, 457-470.

\section{APPENDIX}

The True Distance and the Mean and Standard Deviations of the Distance Estimates, for Each of 78 Distances

\begin{tabular}{|c|c|c|c|}
\hline Segment & $\begin{array}{c}\text { True } \\
\text { Distance }\end{array}$ & $\begin{array}{l}\text { Mean of } \\
\text { Estimates }\end{array}$ & $\begin{array}{c}\text { Standard } \\
\text { Deviations }\end{array}$ \\
\hline $\mathrm{AB}$ & 50.50 & 55.50 & 8.84 \\
\hline $\mathrm{AC}$ & 19.50 & 18.79 & 4.99 \\
\hline $\mathrm{AD}$ & 31.50 & 30.47 & 6.55 \\
\hline $\mathrm{AE}$ & 35.33 & 36.90 & 7.19 \\
\hline $\mathrm{AF}$ & 46.75 & 49.75 & 10.23 \\
\hline $\mathrm{AG}$ & 58.17 & 65.78 & 11.00 \\
\hline $\mathrm{AH}$ & 38.75 & 38.69 & 7.18 \\
\hline AI & 66.00 & 74.21 & 12.59 \\
\hline AJ & 48.50 & 51.24 & 10.12 \\
\hline AK & 48.83 & 58.90 & 10.66 \\
\hline AL & 38.50 & 45.44 & 9.16 \\
\hline AM & 33.33 & 34.61 & 6.93 \\
\hline $\mathrm{BC}$ & 35.33 & 41.81 & 6.01 \\
\hline $\mathrm{BD}$ & 31.33 & 40.22 & 6.94 \\
\hline $\mathrm{BE}$ & 19.83 & 25.02 & 5.29 \\
\hline BF & 32.83 & 45.14 & 8.82 \\
\hline BG & 37.83 & 51.64 & 8.76 \\
\hline $\mathrm{BH}$ & 38.75 & 48.98 & 7.55 \\
\hline BI & 48.67 & 63.92 & 10.10 \\
\hline BJ & 47.83 & 64.17 & 9.72 \\
\hline BK & 65.17 & 85.10 & 10.99 \\
\hline BL & 58.17 & 78.33 & 11.01 \\
\hline $\mathrm{BM}$ & 47.00 & 64.94 & 10.66 \\
\hline $\mathrm{CD}$ & 12.17 & 12.89 & 2.30 \\
\hline $\mathrm{CE}$ & 17.00 & 17.47 & 4.98 \\
\hline CF & 27.50 & 28.76 & 5.91 \\
\hline $\mathrm{CG}$ & 39.00 & 44.17 & 6.99 \\
\hline $\mathrm{CH}$ & 21.17 & 22.83 & 3.66 \\
\hline $\mathrm{CI}$ & 47.50 & 55.24 & 7.59 \\
\hline $\mathrm{CJ}$ & 32.33 & 36.08 & 6.70 \\
\hline
\end{tabular}

\begin{tabular}{lccc}
\hline Segment & $\begin{array}{c}\text { True } \\
\text { Distance }\end{array}$ & $\begin{array}{c}\text { Mean of } \\
\text { Estimates }\end{array}$ & $\begin{array}{c}\text { Standard } \\
\text { Deviations }\end{array}$ \\
\hline CK & 39.67 & 49.30 & 10.63 \\
CL & 30.00 & 42.09 & 9.77 \\
CM & 24.00 & 26.18 & 6.27 \\
DE & 12.17 & 15.60 & 3.06 \\
DF & 15.33 & 15.78 & 2.57 \\
DG & 26.83 & 29.78 & 6.91 \\
DH & 10.50 & 13.08 & 2.61 \\
DI & 35.67 & 37.66 & 6.86 \\
DJ & 22.00 & 27.41 & 5.41 \\
DK & 34.83 & 44.69 & 7.45 \\
DL & 26.83 & 36.08 & 6.86 \\
DM & 15.83 & 19.46 & 4.57 \\
EF & 19.00 & 24.21 & 5.35 \\
EG & 29.00 & 37.71 & 7.17 \\
EH & 20.75 & 27.90 & 4.68 \\
EI & 39.17 & 49.62 & 9.27 \\
EJ & 31.50 & 42.67 & 7.18 \\
EK & 46.50 & 60.42 & 8.31 \\
EL & 38.83 & 53.52 & 8.75 \\
EM & 27.67 & 40.10 & 7.01 \\
FG & 11.50 & 13.15 & 2.92 \\
FH & 11.00 & 19.91 & 5.18 \\
FI & 26.00 & 26.26 & 4.03 \\
FJ & 16.50 & 17.24 & 3.68 \\
FK & 35.00 & 35.97 & 7.21 \\
FL & 31.00 & 38.58 & 8.56 \\
FM & 22.00 & 30.47 & 5.66 \\
GH & 20.83 & 19.19 & 9.90 \\
GI & 10.50 & 12.67 & 2.84 \\
GJ & 19.00 & 23.56 & 5.75 \\
GK & 39.50 & 51.03 & 10.04 \\
GL & 38.37 & 48.66 & 10.00 \\
GM & 31.33 & 42.22 & 7.96 \\
HI & 27.25 & 28.16 & 5.94 \\
HJ & 11.42 & 21.58 & 6.33 \\
HK & 26.33 & 28.92 & 6.46 \\
HL & 20.67 & 24.69 & 7.21 \\
HM & 11.00 & 14.50 & 3.73 \\
IJ & 20.00 & 25.79 & 4.76 \\
IK & 38.83 & 49.93 & 9.31 \\
IL & 46.00 & 55.85 & 10.05 \\
IM & 36.00 & 44.47 & 7.33 \\
JK & 26.00 & 38.76 & 8.04 \\
JL & 19.83 & 30.78 & 8.48 \\
JM & 16.00 & 16.44 & 3.38 \\
KL & 10.50 & 15.82 & 5.54 \\
KM & 19.83 & 23.81 & 4.57 \\
LM & 11.00 & 14.41 & 3.62 \\
\hline & & &
\end{tabular}

(Manuscript received March 2, 1992;

revision accepted for publication January 25, 1993.) 